\title{
Strates
}

STRATES Matériaux pour la recherche en sciences sociales

$12 \mid 2006$

Nouvelles tensions impériales et recompositions en Europe centrale, orientale, et CEI

\section{La transition russe : une interprétation en termes d'incertitude keynésienne}

\section{Anna Kharchenko-Dorbec}

\section{(2) OpenEdition}

\section{Journals}

Édition électronique

URL : http://journals.openedition.org/strates/2372

DOI : $10.4000 /$ strates.2372

ISSN : $1777-5442$

Éditeur

Laboratoire Ladyss

Édition imprimée

Date de publication : 31 décembre 2006

ISSN : 0768-8067

Référence électronique

Anna Kharchenko-Dorbec, «La transition russe : une interprétation en termes d'incertitude keynésienne », Strates [En ligne], 12 | 2006, mis en ligne le 05 septembre 2007, consulté le 08 septembre 2020. URL : http://journals.openedition.org/strates/2372 ; DOI : https://doi.org/10.4000/ strates. 2372

Ce document a été généré automatiquement le 8 septembre 2020.

Tous droits réservés 


\title{
La transition russe : une interprétation en termes d'incertitude keynésienne
}

\author{
Anna Kharchenko-Dorbec
}

Introduction

1 Le système soviétique d'économie planifiée s'est avéré inefficient. En effet, il demandait plus d'information et le traitement important de celle-ci ne fournissait que peu d'incitations à une meilleure productivité. Le passage à l'économie de marché aurait dû aboutir à un dynamisme économique important accompagné par une hausse du niveau de vie de la population. Cependant, l'effondrement des dynamiques économiques observé a été semblable à celui de la seconde guerre mondiale. Les revenus ont chuté, tant en termes de PIB qu'en termes de niveau de vie des ménages (cf. graphique 1). La part des ménages ayant des revenus en dessous du niveau de subsistance avoisine $30 \%$ de la population russe de 1992 à 2000. 
Graphique 1. Dynamique économique de la transition russe 1992-2001

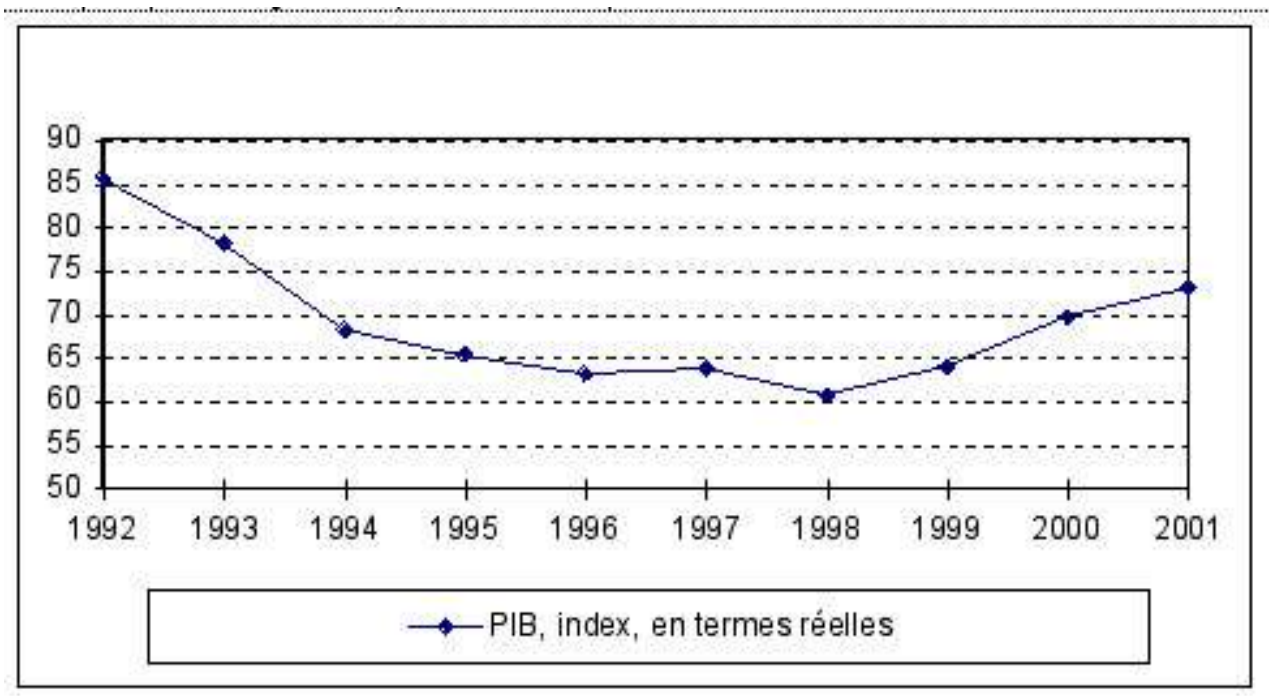

Sour ce: Goskomstat Rossii.

2 D'un point de vue théorique, il faut souligner que la capacité des marchés à s'autoéquilibrer à un point Pareto optimal n'a été démontrée que pour un environnement d'information parfaite (univers parfaitement probabilisable) et symétrique, la présence des imperfections informationnelles aboutissant au mieux à des solutions sous optimales. Selon Joseph E. Stiglitz (1999), la plupart des erreurs commises lors des réformes pendant les dix années de la période de transition auraient pu être évitées si l'on avait porté une plus grande attention aux questions relatives à l'information, concernant par exemple la faillite et la gouvernance de l'entreprise. Ainsi, les travaux empiriques (Stiglitz, 2001) confirment que les pays qui ont connu une privatisation très rapide, sans prêter attention aux questions d'organisation de la gouvernance et de transparence des entreprises nouvellement privatisées, ont eu une croissance significativement affaiblie. Au lieu de créer les conditions de la croissance du bien être social, la privatisation a abouti à la dispersion des actifs et à la destruction de la richesse.

3 Il nous semble que la préférence accordée au scénario libéral sans tenir compte de la construction institutionnelle a été l'une des origines de la longue récession des années 1990 en Russie. La croissance, qui dépend de l'investissement, est liée à l'anticipation des situations futures. Dans un environnement d'incertitude (celui dans lequel l'économie russe s'est retrouvée dès le début des réformes radicales de 1992), en l'absence d'informations suffisantes pour réaliser une évaluation probabiliste du futur, les institutions et les conventions jouent un rôle crucial pour l'ancrage des anticipations collectives. Pour David M. Kreps (1990), l'institution est le résultat des expériences menées à long terme dans une société par des agents à rationalité limitée qui prennent en compte leurs expériences passées. Il est évident que dans la situation de la transition russe, très rapide et radicale, la construction institutionnelle aurait dû faire l'objet d'une politique particulière. De ce fait, l'échec relatif de la transition en Russie et les succès de la Chine, par contraste, ont considérablement amplifié l'importance de la prise en compte du contexte d'incertitude, des problèmes d'information et de la mise en place des institutions, dans l'analyse de la situation. 
L'analyse de la transition russe met en évidence, en effet, l'impossibilité de la décrire en termes de risque probabilisable. Si l'on reprend la définition de G. L. S. Shackle (1955),

par expérience cruciale, je désigne celle où la personne concernée ne peut pas exclure de son esprit que le fait même d'effectuer l'expérience peut détruire pour

toujours les circonstances dans lesquelles elle a été effectuée ${ }^{1}$,

on peut considérer les dynamiques économiques de la période de transition comme cruciales et ainsi impossibles à évaluer en termes de probabilités standard. Dans cette perspective, l'anticipation du futur (une étape nécessaire dans l'évaluation de tout projet d'investissement ayant ainsi un rôle déterminant pour sa dynamique) ne peut pas se baser sur le calcul d'utilité subjective telle qu'elle a été définie par Leonard J. Savage (1954) d'une part, parce que les probabilités des événements déterminant les utilités futures ne sont pas connues (la connaissance exhaustive de ces mêmes événements ne semble pas évidente); d'autre part, l'anticipation de comportement des autres agents économiques joue un rôle déterminant dans les dynamiques futures. Puisqu'en règle générale, les stratégies des autres agents ne sont pas connues, l'anticipation de leur comportement se fait en fonction des éléments de comportements répétitifs et communément admis : les conventions. La construction institutionnelle sert à expliciter ces éléments et à assurer leur répétitivité, devenant ainsi un facteur important de l'ancrage des anticipations.

5 Dans la première partie, on détaillera certaines dynamiques économiques accompagnant le processus de réformes de la période de transition russe. Ensuite on présentera le concept d'incertitude radicale. La troisième partie portera sur les éléments d'analyse de la situation d'incertitude radicale propre à la transition russe en termes de théorie des conventions et d'approche des institutions.

Crise économique et transition

6 Dans la décennie 1990, la Russie est passée par un changement radical de son système économique : en une période extrêmement courte, le rôle de l'État est passé du contrôle total de la vie économique (planification, statut de la propriété) à son retrait au profit du modèle du marché dans sa conception la plus libérale. C'est désormais aux marchés qu'appartient le rôle de moteur des réformes structurelles et de la croissance.

7 Il est important de noter que l'économie planifiée offrait aux entreprises une sécurité exceptionnelle. Étant propriété de l'État, elles n'avaient pas à se préoccuper de leurs ventes et du remboursement de leurs arriérés de paiement ou de leurs crédits, tout était assuré par le plan et une garantie implicite de refinancement assurée par la Gosbank. On pouvait alors parler de la certitude et de la prévisibilité du futur (demande, recouvrement des dettes) au niveau micro-économique. L'arrivée du marché a perturbé cette situation: le marché implique par définition des éléments d'incertitude et une nécessité de former les anticipations sur la demande future et sur la qualité financière des contreparties. Celle-ci peut, dans certains cas, être probabilisable et alors analysée en termes de risque (Tobin, 1956). Si ces éléments ne sont pas probabilisables, on est alors face à l'incertitude pure (Knight, 1921).

Dans cette situation, le problème du remboursement des prêts devient important, le système bancaire à deux niveaux faisant apparaître une véritable contrainte budgétaire.

9 Pour l'analyse de la transition, il est important de tenir compte du fait que l'économie planifiée (la situation de départ) a été une économie dite a-monétaire. Les entreprises fonctionnaient dans les conditions d'une "contrainte budgétaire lâche " (Kornai, 
1999) : dans le cadre du plan, les demandes de fonds de la part des entreprises auprès de la Gosbank (la banque unique) avaient toujours été satisfaites. Au niveau macroéconomique, la banque unique signifie l'égalité automatique entre l'actif et le passif de la Gosbank, la contrainte du système n'est donc pas la contrainte monétaire, mais la contrainte de ressources matérielles. L'économie de marché, quant à elle, est par définition une économie monétaire, une économie, contrainte par la monnaie : ce sont les mécanismes monétaires qui assurent le fonctionnement du mécanisme de concurrence.

Les réformes de la transition russe ont été accompagnées par une crise économique exceptionnelle : la chute du PIB en termes réels entre 1992 et 1998 a été de $40 \%$, celle de la production industrielle de $50 \%$ et celle de l'accumulation brute de capital fixe de $86 \%^{2}$. La baisse du niveau de vie de la population fut comparable seulement avec des périodes de guerre. Le niveau de pauvreté est passé de $2 \%$ de la population à plus de $40 \%^{3}$. Il faudrait y ajouter les enjeux de la privatisation, menée dans des délais extrêmement courts et souvent avec le recours à des schémas opaques et sémi-légaux. La crédibilité du droit de propriété acquis par ces moyens, a suscité des craintes tant des dirigeants des entreprises eux-mêmes que de leur environnement, provoquant des comportements rentiers de la part des propriétaires (Sapir, 2000).

11 L'opacité informationnelle des structures ainsi crées fut renforcée par la faiblesse des institutions (Stiglitz, 2001) : la politique antitrust et autres politiques ont été inefficaces dans l'application à la situation russe. Pendant la période 1992-1998, la monnaie n'a pas été capable de jouer son rôle de régulateur: le gonflement des impayés a remplacé « l'assainissement » du marché et la réforme structurelle. La dollarisation témoigne, à son tour, du manque de confiance dans la monnaie de la part des agents économiques.

À l'issue de dix années de transition en Russie, on peut constater que le passage à l'économie du marché à lui seul n'a pas pu être la condition suffisante d'une meilleure efficacité économique. En effet, d'un point de vue théorique, le système d'économie de marché n'aboutit à l'allocation optimale des ressources que si l'on fait l'hypothèse d'un environnement probabilisable et d'une information symétrique. Or, aucune de ces deux hypothèses ne s'est vérifiée. Il semble donc plus approprié d'analyser la transition en ces termes: l'anticipation du futur dans un environnement incertain ne peut pas se baser sur un calcul probabiliste, mais sur l'imagination et la convention. Les conventions et les institutions jouent alors un rôle d'ancrage des anticipations collectives, ces dernières ayant un impact direct sur les variables économiques. Information et incertitude D'un point de vue théorique, on peut distinguer trois situations en matière d'information et de probabilités, que l'on peut schématiser par le diagramme suivant : 


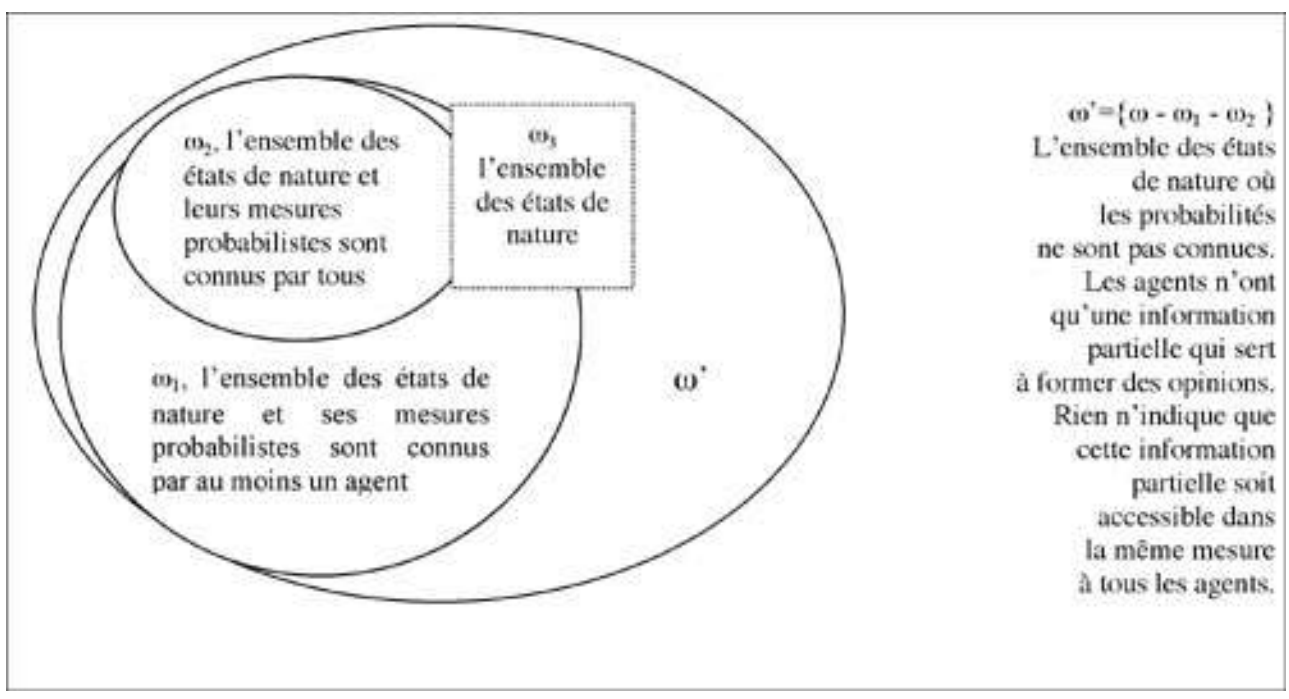

- espace $\omega_{2}$ : la totalité de l'information est connue symétriquement par tous les agents (situation de risque, où les probabilités de tous les événements peuvent être connues par tous les agents).

L'hypothèse de l'information parfaite est essentielle à la réalisation d'un équilibre concurrentiel dans le sens de Kenneth J. Arrow et Gérard Debreu, cet équilibre du marché correspondant à l'optimum de Pareto. Le marché a la capacité de s'autoéquilibrer. L'intervention de l'État doit donc rester limitée. La monnaie est neutre (Friedman, 1971; Sargent, Wallace, 1975): toute politique basée sur la quantité de monnaie n'a pas de conséquences durables en termes réels, mais provoque uniquement l'ajustement de l'inflation. Les choix financiers de l'entreprise sont indifférents (Modigliani, Miller, 1958).

- espace $\omega_{1}$ : information complète (univers probabilisable), mais asymétriquement distribuée entre les agents. Il s'agit des situations où au moins un agent peut connaître la véritable information concernant les dynamiques futures.

Dans la situation d'information imparfaite, son acquisition a un coût qui est assimilable à un coût de transaction (North, 1990). Ainsi, les problèmes de l'aléa moral et de la sélection adverse font apparaître le besoin de signalement et le coût qui lui est associé (Akerlof, 1970). Si l'on adopte l'hypothèse d'information asymétrique, les marchés ne sont plus efficients dans le sens de Pareto. Les phénomènes de rationnement apparaissent (Stiglitz, Weiss, 1981 ; Jaffee, Russell, 1976), ce qui aboutit à des optimum de second rang (Gertler, 1988; Stiglitz, Weiss, 1992). Les marchés ne produisent pas d'incitation pour l'ouverture de l'information : ce rôle est dédié aux institutions au sens large. L'institutionnalisation du traitement de l'information permet aux agents économiques de réaliser des économies importantes. Les institutions bancaires exploitent ainsi un avantage informationnel en matière de crédit (ChevalierFarat, 1992; Bhattacharya, Thakor, 1993). La structure financière de l'entreprise n'est pas neutre, elle est dépendante de la richesse nette et de la liquidité de l'entreprise (Fazzari, Hubbard, Petersen, 1988). L'approche en termes d'asymétrie d'information met en évidence l'existence de la hiérarchie des sources de financement avec une préférence pour l'autofinancement, suivie par le financement par la dette (bancaire et obligataire) et ensuite par l'émission d'actions (Myers, 1984).

- espace $\omega^{\prime}$ : incertitude (Knight, 1921 ; Keynes, 1921 ; Shackle, 1972 ; Minsky, 1986). Les situations d'incertitude se distinguent fondamentalement des situations d'asymétries 
informationnelles par le fait que, dans les situations incertaines, le calcul probabiliste rigoureux n'est pas possible car soit l'ensemble des hypothèses sur les états de nature possibles, soit l'ensemble des mesures probabilistes (probabilités) ne peuvent être connue par aucun agent. L'incertitude se caractérise alors par l'insuffisance de connaissance (Knight, 1921 ; Gilboa, 1989). Pour Paul Davidson (1982-1983), elle serait à l'origine de la non neutralité à long terme de la monnaie.

Les modélisations d'incertitude knightienne en termes de probabilités non-additives (Gilboa, 1989; Gilboa, Schmeidler, 1989; Aizenman, 1995; Eichberger, Kelsey, 2001) limitent la connaissance des probabilités à la connaissance d'un intervalle $\left[p ; p_{\mathrm{u}}\right]$ et non d'une seule valeur/mesure probabiliste. La somme des probabilités ( $p$ ) des événements que l'individu peut envisager est inférieure à l'unité.

Si l'on désigne comme $\gamma$ une mesure de «l'imprécision» de l'évaluation, , the probabilisable additif, alors que $\gamma_{\text {proche }}$ désigne le cas d'incertitude absolue. Les résultats de M. Kilka et M. Weber (2000) démontrent que dans les situations «non familières" les agents ont tendance à avoir un comportement plus prudent en surestimant le pire scénario. Ce type de comportement est souvent appelé le pessimisme ou l'aversion à l'incertitude (Epstein, 1999). Ainsi, pour un agent adverse à l'incertitude, le paramètre $\gamma_{\text {devient }}$ une mesure de cette aversion : la probabilité $\gamma_{\text {est affectée au pire scénario. Une autre }}$ façon d'introduire l'incertitude en termes de probabilités non additives est d'introduire la notion de la contamination des jugements probabilistes (Nishimura, Osaki, 2002; Chen, Epstein, 2002) ${ }^{4}$.

Cependant, il faut remarquer que l'approche en termes de probabilités non additives suppose un univers où l'ensemble des états de nature (des hypothèses) est connu, ce qui représente certainement une limitation de ce type de modèles.

Nous désignerons comme incertitude radicale ou fondamentale les situations, où ni la liste exhaustive des états de nature ni les probabilités ne peuvent être connues. L'importance de la créativité et l'existence des changements structurels imprévisibles distinguent le concept d'incertitude radicale des autres concepts. Il faut souligner que dans des situations radicalement incertaines, une partie de l'information n'existe pas au moment de la décision car le futur est en construction dans le moment présent. En effet, l'incertitude radicale ne peut pas être éliminée ex-ante suite à la découverte d'informations supplémentaires ou à l'apparition d'une nouvelle technologie de traitement de l'information, car, au moment de la décision, l'information en question n'existe pas tout simplement (Knight, 1921 ; Shackle, 1955 ; Davidson, 1991, 1996). Les décisions cruciales et irréversibles sont prises en se basant sur les opinions, intuitions et jugements subjectifs. Il faut préciser que l'information qui permet de former des opinions n'est pas nécessairement symétrique, la fonction de signalement reste donc importante.

18 Cette distinction nous paraît importante dans l'interprétation de l'incertitude propre au processus de la transition en Russie. Les enjeux de la transformation de l'économie russe en une économie de marché n'ont pas pu tous être envisagés ni par les organismes internationaux ni par les agents : aucun agent n'a été donc suffisamment informé pour être en mesure d'anticiper les dynamiques économiques russes. L'incertitude et l'instabilité des anticipations qui lui sont associées sont les causes 
principales de la chute de l'investissement, et ainsi la clé de l'instabilité macroéconomique générale.

Le concept de Shackle (1969), (1972) se base sur le caractère crucial et irréversible des décisions prises dans l'environnement incertain. Ce type d'expérience logiquement ne peut être reproduit en série et doit ainsi être traité comme une expérience isolée et non reproductible. Même dans la situation où on peut logiquement imaginer la répétition de l'expérience, il faut toujours savoir qu'au moment d'une nouvelle réalisation les circonstances seront définitivement modifiées. Le calcul des probabilités des expériences cruciales n'est donc pas possible : leurs dynamiques sont caractérisées par la surprise potentielle et la non-ergodicité 5 . Cependant, le calcul probabiliste peut être possible dans le cas des décisions de " routine » concernant des expériences divisibles ${ }^{6}$.

L'existence des fonctions de distribution (mesures probabilistes) déterminées signifierait, selon G. L. S. Shackle (1972), que le futur soit connu à l'avance et qu'il ne reste plus qu'à le voir venir : «Le monde de prévision parfaite devrait être sans temps et sans événement ${ }^{7}$. " Dans cette situation il est inutile de parler de choix. Le choix pour G.L.S. Shackle est irréversible et crucial, il va être un facteur déterminant pour les situations futures. Il faudrait souligner ce caractère historique de l'approche shackelienne: tout événement de la vie économique représente une source de changements successifs qui s'inscrit dans le temps historique.

21 Pour P. Davidson (1996), la nonergodicité est une caractéristique fondamentale du monde économique. En rejoignant G.L.S.Shackle, P.Davidson affirme que l'environnement objectivement probabilisable signifie que tous les changements futurs doivent être prédéterminés par des paramètres fondamentaux. Un tel système s'avère impossible à changer par une action humaine, cette dernière devenant alors un outil mécanique et non une véritable décision.

La non-stationnarité est une condition suffisante de la non-ergodicité : dans un modèle non stationnaire les agents ne peuvent pas prédire le futur de façon fiable en se basant sur les séries temporelles passées, les changements non programmées des lois probabilistes déterminant les dynamiques des séries économiques sont envisageables. En effet, le caractère crucial du choix économique est à l'origine de cette nonergodicité : rien n'indique que la loi probabiliste avant et après le choix crucial soit la même.

Robert P. Flood et Peter M. Garber (1994) distinguent les périodes «cruciales » des périodes de "routine" où le comportement peut posséder les caractéristiques de prédictibilité et d'ergodicité. La décision cruciale déplace le système dans une autre position ou un autre régime de façon imprédictible.

G. L. S. Shackle souligne à plusieurs reprises que la liste des hypothèses dont l'individu dispose n'est pas exhaustive: "[...] les conséquences possibles de toute action ne constituent pas, en général, un ensemble fini et limité $[. .$.$] ». Dans cette situation, le$ simple choix d'une des hypothèses se transforme en un processus créatif : l'individu doit imaginer des hypothèses nouvelles ${ }^{9}$. La liberté de choix présuppose par définition, l'impossibilité de connaître le comportement des autres et ainsi de l'intégrer dans l'anticipation. La liberté de l'individu rend impossible la prévision des interactions sociales. Cependant, cette liberté de choix représente une source supplémentaire de connaissance imparfaite et de ce fait, d'incertitude. 
Le développement récent de la théorie shackelienne s'inscrit dans le cadre de la théorie de l'évidence (Shafer, 1976 ; Fioretti, 2001).

L'ensemble de l'évidence empirique est supposé décrit en nombres $\left[m\left(A_{A}\right), m\left(A_{2}\right), m\left(A_{A}\right) \ldots\right]$, qui représentent l'évidence qui supporte les hypothèses $\left[A_{1}, A_{0}, A_{3} \ldots\right] ;$ ie nombre $m(\theta)$, affecté au jugement $\Theta$ représente le fait que l'ensemble des hypothèses $\left\{A_{1}, A_{2}, A_{3} \ldots\right\}$ n'est pas exhaustif. Chaque ensemble $\left\{\mathrm{m}_{1}, \mathrm{~m}_{2}, \mathrm{~m}_{3} . ..\right\}$ désigne un corps de l'évidence : un corps de l'évidence est associé à l'ensemble des hypothèses $\left\{A_{1}, A_{2}, A_{3} \ldots \Theta\right\}$, un autre à $\left\{B_{1}, B_{2}, B_{3} \ldots \Theta\right\}$, etc.,

$\sum m(()+,m(\theta)-1$

de même que

$\sum m\left(B_{1}\right)+m(\theta)-1$ etc.

Les appréciations individuelles sont ensuite présentées comme des fonctions de croyance. Notons que la présence de $m(\theta)$ suppose que la somme des évidences connues est inférieure à l'unité, ce qui rejoint le concept des probabilités subadditives. Cependant, la présence de $\Theta$ démontre bien l'inexhaustivité de l'ensemble des hypothèses. L'apparition de nouvelles hypothèses est également possible : elle signifie l'apparition de nouveau corps de l'évidence sans affecter les corps de l'évidence précédents, ce qui rejoint le raisonnement schackelien.

Dans la théorie keynésienne ${ }^{10}$, dans un environnement d'incertitude, la décision individuelle est influencée par le comportement (ou, plus exactement, l'anticipation de comportement) des autres individus. L'anticipation du comportement des autres individus devient une variable de commande dans la prise de décision dans un environnement d'incertitude keynésienne. La décision et l'anticipation prennent ainsi une ampleur sociale: dans un monde keynésien, l'anticipation est inconcevable en dehors de la société (marché, autres agents).

8 John M. Keynes (1937) distingue deux éléments essentiels dans le processus d'anticipation des situations fondamentalement incertaines. Le premier consiste à étudier les états précédents pour définir la base pour l'extrapolation. En effet, si le prêteur veut savoir si son futur crédit sera remboursé, il s'intéressera en premier lieu à l'historique du crédit de l'emprunteur. Cette attitude extrapolative permet de donner une première approximation des pronostics de courte période. Il est évident, que l'extrapolation pure n'est opérationnelle que dans un monde certain, voire stationnaire. Dans une situation incertaine et instable où la croyance partagée dans le maintien des tendances passées est faible, l'attitude extrapolative à elle seule n'est pas suffisante : il est indispensable d'imaginer les états futurs (d'imaginer, car pour les situations d'incertitude elles ne peuvent pas être connues et donc calculées). Pour un agent économique qui fait partie de la société, l'anticipation des états futurs devient indissociable de l'anticipation de comportement des autres agents parce que ce comportement influe sur la situation future.

Il faut souligner que si la croyance dans le maintien des tendances passées est faible, ce qui est le cas de la période très courte propre à la transition, avec toutes les entreprises nouvellement créées opérant dans un environnement en changement continu, l'anticipation sera grandement influencée par l'opinion sur l'évolution future. Puisque l'information exhaustive sur les changements futurs n'est pas disponible, il suffit que la confiance dans les jugements individuels ne soit pas absolue, pour que les entrepreneurs aient intérêt à se référer au jugement des autres qui sont peut-être 
mieux informés ${ }^{11}$. Cette croyance dans la meilleure information des autres représente le cas idéal d'un jugement conventionnel ${ }^{12}$.

30 Ainsi, le comportement d'un agent individuel qui aurait une faible confiance dans le maintien des tendances passées, ainsi que dans ses propres estimations, devient plus conforme au comportement de la majorité (et ainsi favorable à la spéculation). L'interdépendance entre les agents prend une ampleur particulière : le résultat d'une décision d'un agent dépend de la décision prise par les autres. L'interdépendance devrait être combinée avec la possibilité de comportement créatif des individus. Le fait que les anticipations se basent entre autres sur l'estimation de l'anticipation de l'autre renforce l'incertitude fondamentale.

31 Dans ces conditions, la monnaie perd sa neutralité (Keynes, 1973 ; Minsky, 1991; Davidson, 1982-1983). En effet, dans le système keynésien, sa demande est régie par la préférence pour la liquidité et par le motif de finance. Ces variables ne dépendent pas uniquement des variables réelles, mais aussi des anticipations et sont ainsi affectées par l'incertitude.

Conventions et institutionsApproche en termes de convention

Puisqu'il est difficile d'imaginer ce qu'imaginera l'autre, il peut être utile de se référer à des éléments du comportement des agents dont il est communément admis qu'ils se reproduiront, ce que la théorie récente désigne comme convention. L'imprévisibilité du futur fait apparaitre un gain de coordination entre les agents non altruistes: la convention apparaît comme une solution à la coordination des actions individuelles au sein d'une action collective impliquant des intérêts communs (Lewis, 1969). Elle représente également un moyen (peu coûteux) d'acquérir l'information sur l'expérience antérieure et donne les éléments de la stratégie future des autres agents (Favereau, 1998). Elle devient ainsi un instrument d'ancrage des anticipations (Mc Adams, 2001).

33 La convention est une règle particulière qui coordonne les comportements avec régularité. On peut dégager quatre caractéristiques de celle-ci (Batifoulier, 2001) s'appuyant notamment sur les travaux de Lewis et Leibenstein :

34 - elle est arbitraire : d'autres possibilités à se coordonner semblent possibles ;

35 - elle n'a pas besoin de sanctions explicites en cas de non-respect, mais une menace implicite peut exister ;

36 - même si parfois il est possible d'en donner une énonciation explicite, il n'existe pas de formulation officielle de la convention ;

37 - la convention, lorsqu'elle débouche sur une coordination réussie, suspend l'explication des raisons d'agir. Dans ce cas, elle ne nécessite pas de justifications précises. Le renouvellement de la justification à chaque tour du jeu n'est donc pas nécessaire. Par conséquent, l'évolution de la convention n'est pas évidente et ne se fait pas uniquement par rapport au raisonnement de Pareto efficacité.

38 Concernant la transition russe, le passage d'une économie planifiée à une économie de marché a fait changer radicalement les conventions sur lesquelles se basait tout comportement économique. Cet aspect a été négligé lors de la phase «libérale » des réformes : aucune politique en matière d'information et d'ancrage des anticipations n'a été menée (Renversez, 2001). Dans cette situation, les agents économiques ont adopté un comportement adverse à l'incertitude en se référant au " pire scénario ». 
39 Ainsi, vis-à-vis de la monnaie, ce comportement se manifeste par une très forte dollarisation : le dollar joue le rôle de l'unité de compte pour les agents économiques russes. L'épargne des ménages russes reste également libellée en devises étrangères (et principalement en dollars US), les devises étrangères détiennent la première place parmi les placements des ménages (autour de $6 \%$ du revenu disponible en 2001-2002, la part des dépôts bancaires demeurant à environ $4 \%$ pour la même période). Concernant les dépôts bancaires, $40 \%$ environ sont libellés en devises.

40 Au sein des entreprises, le comportement adverse à l'incertitude demeure également à l'origine du rôle très limité du financement externe, ce dernier étant majoritairement représenté par le financement public $(20 \%$ du financement de l'investissement en 2001). Le crédit bancaire ${ }^{13}$ sert majoritairement à financer les fonds de roulement des entreprises ${ }^{14}$. L'essentiel du financement de l'économie est fait sur ressources propres : $50 \%$ de l'investissement en 2001. Le marché des capitaux reste dominé par quelques entreprises de taille géante - monopoles naturels ou appartenant au secteur des matières premières - (Gazprom, RAO EES, Sibneft, etc.), les autres entreprises n'étant pas en mesure de fournir des signaux suffisamment crédibles pour pouvoir lever les fonds du public. Il apparaît donc que la majorité des entreprises ne peut compter que sur les fonds propres.

41 Il faut remarquer, que, comme toute convention, cette aversion à l'incertitude manifeste des signes de persistance. Elle continue à régir le comportement des agents aujourd'hui, même si les incertitudes économiques semblent se réduire ${ }^{15}$.

Institutions et transition

42 En présence d'incertitude, les institutions apparaissent comme un moyen d'expliciter et de renforcer le comportement conventionnel. La prise en compte des institutions introduit un aspect social dans l'échange économique. Les institutions jouent un rôle important car elles représentent un moyen de rendre publique l'information sur le comportement des autres, de réduire les asymétries informationnelles (rappelons que ces asymétries sont également propres à des situations d'incertitude), ainsi que de renforcer les contrats dans des situations de l'aléa moral (Stiglitz, 1998; Aoki, 2000 ; North, 1990).

43 P. Davidson (1982-1983) insiste sur le rôle des institutions en tant que réductrices d'incertitude. Dans un monde économique non ergodique, les politiques doivent continuellement s'adapter à des changements dans l'environnement. Le rôle du gouvernement consiste à adapter le système institutionnel à ce changement continu et imprédictible.

44 Le concept d'efficience adaptative de North (2003) semble rejoindre celui de Davidson. Dans un monde non ergodique, le système institutionnel (qui comprend trois composantes: les règles formelles, les règles informelles et les procédures de renforcement) doit pouvoir s'adapter de façon efficace aux changements du monde extérieur. Un mécanisme de feedback (les retours de l'information sur les changements de la vie réelle vers les institutions) joue un rôle crucial dans un tel système institutionnel. Il faut remarquer que les changements des règles formelles sont les seuls possibles à court terme. La dynamique des règles informelles s'inscrit dans la logique des croyances et des conventions, son évolution devient une affaire à plus long terme. Si l'on décrit la société en termes de jeu évolutif, pour North (1990) les institutions représentent des règles du jeu exogènes par rapport aux joueurs. Elles représentent des 
contraintes qui déterminent l'interaction entre les individus en limitant l'ensemble des choix individuels (ayant ainsi un impact sur le système des incitations). En ce qui concerne les procédures de renforcement, l'analyse en termes d'équilibre de Nash démontre (Hurwicz, 1996) que toutes les structures institutionnelles ne peuvent pas être renforçables. Si l'une des structures ou politiques publiques se révèlent incompatibles avec les intérêts des joueurs (les joueurs sont incités à utiliser une stratégie ne s'inscrivant pas dans l'ensemble prédéfini par les règles formelles), les règles ne sont pas autorenforçables et donc cette politique peut ne pas être soutenable. L'apparition du marché noir suite à des restrictions commerciales excessives en est un très bon exemple.

Masahiko Aoki (2000) décrit l'économie en termes de jeu cognitif où les agents n'ont pas nécessairement d'information complète concernant le jeu en question et les stratégies des autres. Ils ont des visions individuelles, incomplètes et cognitives. Les règles du jeu sont endogènes : "[...] elles sont créées de façon endogène suite à des interactions stratégiques, maintenues dans les esprits des agents et ainsi autoentretenues ${ }^{16} »$. L'institution apparaît comme une composante commune de ce jeu subjectif, une croyance partagée et autoentretenue concernant le jeu joué ${ }^{17}$. L'équilibre du système est endogène et apparaît alors comme une réalité socialement construite. À l'équilibre, les croyances des agents sont coordonnées via leurs représentations sommaires et symboliques. L'analyse en termes d'équilibre met en évidence une double nature de l'institution chez M. Aoki, son endogénéité et son objectivité.

Lorsque l'équilibre du jeu diffère sensiblement de celui attendu par les agents, on se trouve en situation de crise cognitive générale, les agents sont poussés à la recherche de nouveaux modèles subjectifs, ce qui aboutit à un nouvel équilibre. Des études empiriques confirment une grande instabilité du cadre institutionnel russe. Ainsi, l'étude de Kisunko, Benetti et Weder (1997) ${ }^{18}$ menée sur un large panel d'entreprises de plusieurs pays en transition (vingt pays, l'échantillon incluant la Russie) démontre que l'environnement légal et institutionnel dans la majorité des pays en transition a été caractérisé par une très forte imprévisibilité. Ainsi, les entreprises de la région incluant la Russie, l'Ukraine, la Biélorussie et la Moldavie (223 entreprises au total, l'étude porte sur la période 1996-1997) rapportent une forte imprévisibilité de changement des règles et des politiques $(83,9 \%$ des entreprises considèrent des politiques publiques comme imprédictibles dont 19,6\% comme complètement imprévisible). La situation avec la prévisibilité de l'environnement légal a également empiré durant la phase de transition pour $46,3 \%$ des entreprises (elle s'est améliorée pour $9 \%$ des entreprises seulement). Ainsi, $78 \%$ des entreprises de la région qui inclut la Russie indiquent que l'imprévisibilité de l'environnement légal représente un obstacle à l'activité économique de leur entreprise. L'étude observe également une faible crédibilité des politiques publiques ( $78,5 \%$ des entrepreneurs la considèrent comme faible dont $10,4 \%$ répondent que le gouvernement n'atteint jamais des objectifs annoncées) ainsi qu'une faible transparence de ces politiques $(79,1 \%$ des entreprises rapportent un manque de l'information sur les mesures de la politique publique mise en place dont $15,7 \%$ de l'absence totale d'une telle information). Cependant, les entreprises signalent un impact fort des mesures de la politique publique sur l'activité économique de leur entreprise ( $82 \%$ dont l'impact est direct pour $29,6 \%$ ). L'étude indique également qu'une instabilité politique telle qu'on l'observe durant la transition, qu'il s'agisse de changements constitutionnels ou de crises imprévues, a un impact considérable sur 
l'environnement des affaires (73\% des entreprises subissent des conséquences significatives de ces changements). révision coordonnée des croyances collectives suite à des chocs (technologiques, politiques).

\section{Conclusion}

La période de transition russe et l'incertitude radicale qui l'a accompagnée ont donc été à l'origine de la formation des comportements conventionnels marqués par une très forte aversion à l'incertitude, aboutissant à une généralisation des comportements rentiers.

50 Le fait que la construction institutionnelle n'ait pas été considérée comme prioritaire lors des réformes de la transition a abouti à des déformations du système de coordination des anticipations collectives. Cette déformation s'est transcrite en une conventionnalisation du comportement d'aversion à l'incertitude, dont la dollarisation, la fuite des capitaux et la faiblesse du secteur financier représentent d'importants exemples. En effet, les conventions de l'économie du marché ont été définies en se basant sur la faible crédibilité et l'instabilité des institutions. La faiblesse des institutions et l'absence d'une politique spécifique orientée vers la réduction de l'incertitude et l'ancrage des anticipations collectives ne permet pas à l'heure actuelle de combler de nombreux retards, en particulier en matière de financement de l'investissement.

51 Cependant, la construction institutionnelle représente l'une des conditions nécessaires à la réduction de l'incertitude et à la mise en place de nouvelles conventions permettant d'assurer une croissance stable. Cette réduction semble se heurter encore aujourd'hui à des comportements désormais conventionnés adoptés lors de phase libérale de la transition.

\section{BIBLIOGRAPHIE}

Aizenmann J., 1995, « Investment in new Activities and the Welfare Cost of Uncertainty », NBER Working Paper, ${ }^{\circ}$ 5041, February.

Akerlof G., 1970, «The Market for Lemons: Quality Uncertainty and the Price Mecanism », Quaterly Journal of Economics, vol. 84, August, p. 488-500.

Aoki M., 2000, « What are Institutions and How Should We Approach Them? », Villa Borsig Workshop Series, The Institutional Foundations of a Market Economy.

Batifoulier P. (ed.), 2001, Théorie des conventions, Paris, Economica, collection Forum.

Bhattacharya S., Thakor A. V., 1993, « Contemporary Banking Theory », Journal of Financial Intermediation, $\mathrm{n}^{\circ} 3$, p. 2-50.

Chen Z., Epstein L., 2002, « Ambiguity, Risk and Asset Returns in Continuous Time », Econometrica, $\mathrm{n}^{\circ} 70$, p. 1403-1443.

Strates, $12 \mid 2006$ 
Chevalier-Farat T., 1992, « Pourquoi des banques », Revue d'Économie politique, Septembre-Octobre, p. 633-685.

Davidson P., 1982-1983, « Rational Expectations: A Fallacious Foundation for Studying Crucial Decision-Making Processes », Journal of Post-Keynesian Economics, vol. 5, n² 2, Winter, p. 182-198.

Davidson P., 1991, « Is Probability Theory Relevant for Choice under Uncertainty? », Journal of Economic Perspectives, 5(1), p. 129-143.

Davidson P., 1996, « Reality and Economic Theory », Journal of Post-Keynesian Economics, vol. 18, $n^{\circ} 4$, p. $479-508$.

Dorbec A., Renversez F., 2003, « Écart de la composante réelle et de la composante monétaire dans les relations économiques russo-européennes ", Paris, contribution au colloque franco-russe Problèmes monétaires et financiers de la transition en Russie.

Eichberger J., Kelsey D., 2001 « Uncertainty and Strategic Interaction in Economics », Journal of Economic Issues.

Epstein L., 1999, « A Definition of Uncertainty Aversion », Review of Economic Studies, n 66, p. 579-608.

Favereau O., 1994, « Règle, organisation et apprentissage collectif : un paradigme non standard pour trois théories hétérodoxes ", in Orléan A. (ed.), Analyse économique des conventions, Paris, Puf, p. 113-137.

Favereau O., 1997, « L'incomplétude n'est pas le problème, c'est la solution », in Reynaud B. (ed.), Les limites de la rationalité, t. $2:$ Les figures du collectif, Paris, La Découverte, p. 219-234.

Favereau 0. 1998, «L'économie normative de la rationalité limitée », in Brochier H. (ed.), L'économie normative, Paris, Economica, p. 109-123.

Fazzari S., Hubbard R., Petersen B., 1988, « Financing Constraints and Corporate Investment », Brookings Papers of Economic Activity, vol. 1, p. 351-369.

Fioretti G., 2001 , « A mathematical theory of Evidence for G.L.S. Shackle », ISER seminar series.

Flood R., Garber P., 1994, Speculative Bubbles, Speculative Attacks, and Policy Switching, Cambridge, MIT Press.

Friedman M., 1971, « La théorie quantitative de la monnaie. Une nouvelle présentation », in Thorn R., Théorie monétaire, Paris, Dunod.

Gertler M., 1988, «Financial Structure and Aggregate Economic Activity: an Overview », NBER Working Paper, $\mathrm{n}^{\circ} 2559$.

Gilboa I., 1987, « Expected Utility with Purely Subjective Non-Additive Probabilities », Journal of Mathematical Economics, $n^{\circ} 16$, p. 65-88.

Gilboa I., Schmeidler D., 1989, « Maxmin Expected Utility with Non-Unique Prior », Journal of Mathematical Economics, $n^{\circ}$ 18, p. 141-153.

Jaffee D. M., Russell T., 1976, « Imperfect information, Uncertainty and Credit Rationing », Quaterly Journal of Economics, vol. 90, November, p. 651-666.

Keynes J. M., 1921, Treatise on Probability, Collected Writings, vol. VIII. New York, St. Martin's Press, 1973.

Keynes J. M., 1936, The General Theory of Employment, Interest and Money, London, Macmillan, Collected Writings, vol. VII, New York, St. Martins Press, 1973. 
Keynes J. M. 1973, Collected Writings, vol. IX, vol. XX, vol. XII, New York, St. Martin's Press.

Keynes J. M., 1937, « The Theory of the Rate of Interest », The General Theory and After, Collected Writings, vol. XIV.

Kilka M., Weber M., 2000, «What Determines the Shape of the Probability Weighting Function under Uncertainty? », Working Paper, Universität Mannheim.

Knight F., 1921, Risk, Uncertainty and Profit, New York, Houghton Miffin.

Kornaï J., 1999, " Du socialisme au capitalisme : la signification du "changement de système" ", in Chavance B. et al. (eds), Capitalisme et socialisme en perspective. Évolution et transformation des systèmes économiques, Paris, La Découverte, p. 317-347.

Kornaï J., 2001, « Dix ans après The Road to a Free Economy : l'auto-évaluation de l'auteur », in La transformation postsocialiste. Dilemmes et décisions, Paris, Éditions de la Maison des sciences de l'homme, p. 263-278.

Kreps D., 1990, A course in microeconomic theory, New York, Harvester Wheatsheaf.

Leibenstein H., 1984, « On the Economics of Conventions and Institutions: an Exploratory Essay », Journal of Institutional and Theoretical Economics, $n^{\circ} 140$, p. 74-86.

Leibenstein H., 1984, Inside the firm, Harvard University Press.

Lewis D., 1969, Convention. A philosophical Study, Cambridge University Press.

Mc Adams, 2001, « Conventions and Norms (Philosophical aspects) » in Smelser N., Bates P. (eds.), International Encyclopedia of of the social and behavioural sciences.

Minsky H. P., 1986, Stabilizing an Unstable Economy, Yale University Press.

Minsky H. P., 1991, « The Endogeneity of Money », in Nell E., Semmler W. (eds.), Kaldor and Mainstream Economics, Macmillan.

Modigliani F., Miller M., 1958, « The Cost of Capital Corporate Finance and the Theory of Investment », American Economic Review, vol. XLVIII, June, nº 3, p. 261-297.

Myers S., 1984, « The Capital Structure Puzzle », Journal of Finance, vol. 39, July, p. 575-592.

Nishimura K., Ozaki H., 2002, « Irreversible investment and knightian uncertainty », ISER Seminar Series 3/5/03.

North D. C., 1990, Institutions, Institutional Change and Economic Performance, Cambridge University Press.

North D. C., 2003, Understanding the Process of Economic Change, conférence faite à l'European School on New Institutionnal Economies.

Renversez F., 2001 « La transition inaboutie du système financier en Russie ", Diogène, n 194, Recomposition des espaces post-comunistes, avril-juin, Unesco, Paris.

Renversez F., Kharchenko-Dorbec A., 2002, « Evaluation of Influence of Financial Factors to the recent Economic Growth in Russia », Studies of Russian Economic Development, $\mathrm{n}^{\circ} 3$.

Rosser J. B, Kramer K. L., 2001, « Alternative Keynesian and Post Keynesian Perspective on Uncertainty and Expectations », Journal of Post Keynesian Economics, Summer 2001, vol. 23, $\mathrm{n}^{\circ} 4$, p. 545-566.

Sapir J., 2000, « La crise financière russe d'août 1998, tournant de la transition en Russie ? ", document de travail 01-1, CEMI, EHESS. 
Sargent T., Wallace N., 1975, « Rational Expectations, the Optimal Monetary Instrument and the Optimal Money Supply Rule », Journal of Political Economy, vol. 83, n² 2, April, p. 241-254.

Savage L. J., 1954, The Foundations of Statistics, New York, Wiley.

Schmeidler D., 1989. « Subjective Probability and Expected Utility without Additivity », Econometrica, vol. 57, p. 571-87.

Shackle G. L. S., 1955, Uncertainty in Economics and other Reflexions, Cambridge University Press. Shackle G. L. S., 1969, Decision order and time in human affairs, Cambridge University Press.

Shackle G. L. S., 1972, Epistemics \& Economics: a critique of economic doctrines, Cambridge University Press.

Shafer G., 1976, A Mathematical Theory of Evidence, Princeton University Press.

Stiglitz J. E., 1998, « More Instruments and Broader Goals: Moving Toward the Post-Washington Consensus ", The 1998 Wider Annual Lecture, Helsinki, January.

Stiglitz J. E., 2000, « Whither Reform? Ten Years of the Transition », Proceedings of the Annual Bank Conference on Development Economics 1999, Washington, D.C., World Bank, p. 27-56.

Stiglitz J. E., 2001, « Quis Custodiet Ipsos Custodes? », in Stiglitz J. E., Muet P. A. (eds.), Governance, equity, and global markets: the Annual Bank Conference on Development Economics, Europe, World Bank, New York, Oxford University Press, p. 22-54.

Stiglitz J. E., Weiss A., 1981, « Credit Rationing in Markets with Imperfect Information », American Economic Review, 71(3), June, p. 393-410.

Stiglitz J. E., Weiss A., 1983, « Alternative Approaches to the Analysis of Markets with Asymmetric Information », American Economic Review, 73(1), March, p. 246-249.

Stiglitz J. E., Weiss A., 1986, « Credit Rationing and Collateral » in J. Edwards et al. (eds.), Recent Developments in Corporate Finance, New York, Cambridge University Press, p. 101-135.

Stiglitz J. E., Weiss A., 1992, « Asymmetric Information in Credit Markets and Its Implications for Macro-economics », Oxford Economic Papers, 44(4), October, p. 694-724.

Tobin J., 1956, « Liquidity Preference as the Behaviour towards Risk », Review of Economic Studies, August.

\section{NOTES}

1. [By a crucial experience I mean one where one person concerned cannot exclude from his mind the possibility that the very act of performing the experience may destroy for ever the circumstances in which it was performed], Shackle G. L. S., 1955.

2. Données Goskomstat.

3. Estimations de la Banque mondiale.

4. En règle générale, si $\mathrm{M}$ est un ensemble de probabilités associées à des événements

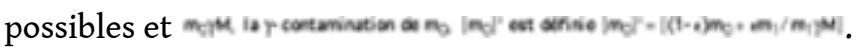

5. Le processus stochastique est ergodique, si, pour un nombre de réalisations infini, les statistiques spatiales et temporaires convergent. Pour une série ergodique, les statistiques temporaires et spatiales calculées en se basant sur des données empiriques passées peuvent être considérées comme des estimations sans biais des distributions probabilistes objectives permettant une évaluation de la réalisation de la même série à 
une date future donnée. On peut alors parler d'une équivalence en termes de probabilités entre les données passées et les données futures : elles sont décrites par la même loi probabiliste.

6. Pour la définition de l'expérience divisible, voir, par exemple, Shackle G.L.S., 1955, Uncertainty in Economics, chapitre I.

7. [The world of perfect foresight would be timeless and event-less], Shackle, 1972, p. 165.

8. [... the possible outcomes of any act do not, in general, constitute a limited and a finite set...], Shackle, 1969 , p. 7.

9. Shackle, 1955, p. 74, «I conceive the nature of expectation itself as an act of creative imagination and not of "rational" calculation; for calculation is impossible when the data are incomplete and in face of ignorance, rationality is a mere pretence [Je conçois la nature de l'anticipation elle-même en tant qu'un acte de l'imagination créative et non de calcul rationnel ; car le calcul est impossible lorsque les données sont incomplètes et en face de l'ignorance, la rationalité n'est rien qu'une prétention] »

10. Keynes J. M., 1936.

11. [...fall back on the judgment of the rest of the world which is perhaps better informed], J. M. Keynes, 1937.

12. [...the psychology of a society each of whom is endeavoring to copy the others leads to what we strictly term a conventional judgment ], J. M. Keynes, 1937, p. 114.

13. En 2001-2003, $55 \%$ du crédit bancaire est octroyé en devises et principalement en dollars US.

14. Renversez F., Kharchenko-Dorbec A., 2002.

15. Dorbec A., Renversez F., 2003.

16. "We do regard them [rules of the game as being endogenously created through the strategic interactions of agents, held in the minds of agents, and thus self-sustaining ", Aoki M., 2000. 17. "...institution as a self-sustaining system of shared beliefs... », Aoki M., 2000.

18. Brunetti A. Kisunko G., Weder B., 1997 : « Institutions in Transition: Reliability of Rules and Economic Performance in Former Socialist Countries », World Bank Policy Research Working Paper.

\section{RÉSUMÉS}

Cet article propose une interprétation théorique de la transition en Russie en termes d'incertitude keynésienne. Cette trame théorique souligne la nécessité de politiques d'ancrage claires des attentes des acteurs. Celles-ci sont spécialement importantes pour la construction de systèmes financiers capables de susciter un développement durable. Les difficultés actuelles de satisfaire ces attentes qui tiennent à la profonde transformation du système économique en Russie et à l'absence de politiques institutionnelles claires ont entraîné une récession en incitant les acteurs à adopter une attitude de non coopération.

Anna Kharchenko-Dorbec. The transition in Russia: an interpretation in terms of keynesian uncertainty This paper suggests a theoretical interpretation of problems during the transition in Russia in terms of Keynesian uncertainty. This theoretical framework suggests the necessity for clear policies of anchoring expectations especially important for the construction of financial systems 
capable of promoting a sustainable growth. The existing difficulties in making expectations relate to a deep transformation of the economic system in Russia and in the absence of clear institutional policies, have enforced the economic recession by inciting agents to adopt a noncooperative behaviour.

\section{AUTEUR}

\section{ANNA KHARCHENKO-DORBEC}

ATER, Université Paris X-Nanterre, Modem/CNRS, adorbec@u-paris10.fr 